\title{
A Variant of Fermat's Diophantine Equation
}

\author{
Serdar Beji \\ Faculty of Naval Architecture and Ocean Engineering, Istanbul Technical University, Istanbul, Turkey \\ Email: sbeji@itu.edu.tr
}

How to cite this paper: Beji, S. (2021) A Variant of Fermat's Diophantine Equation. Advances in Pure Mathematics, 11, 929-936. https://doi.org/10.4236/apm.2021.1112059

Received: November 1, 2021

Accepted: December 5, 2021

Published: December 8, 2021

Copyright (c) 2021 by author(s) and Scientific Research Publishing Inc. This work is licensed under the Creative Commons Attribution International License (CC BY 4.0).

http://creativecommons.org/licenses/by/4.0/

\begin{abstract}
A variant of Fermat's last Diophantine equation is proposed by adjusting the number of terms in accord with the power of terms and a theorem describing the solubility conditions is stated. Numerically obtained primitive solutions are presented for several cases with number of terms equal to or greater than powers. Further, geometric representations of solutions for the second and third power equations are devised by recasting the general equation in a form with rational solutions less than unity. Finally, it is suggested to consider negative and complex integers in seeking solutions to Diophantine forms in general.
\end{abstract}

\section{Keywords}

Variant of Fermat's Last Equation, Positive Integer Solutions of New Fermat-Type Equations, Geometric Representations for Solutions of New Diophantine Equations

\section{Introduction}

Diophantus (200-284), famed as the father of algebra, is known for his works on quadratic equations and puzzle-like algebraic problems such as finding numbers satisfying the condition that difference of the cubes of two numbers is equal to the sum of the cubes of two other numbers [1]. Fermat (1601-1665), quite familiar with the works of Diophantus, posed several Diophantine problems, among them the special case of the last theorem that the sum of two cubes cannot be a cube. Concerning the last theorem Fermat's note in the margin of his now lost copy of Diophantus's Arithmetica that "To divide a cube into two other cubes, a fourth power or in general any power whatever into two powers of the same denomination above the second is impossible, and I have assuredly found an admirable proof of this, but the margin is too narrow to contain it" has unquestionably been the most firing remark giving rise to hopes for a relatively simple 
proof [1]. Thus, regardless of the complete rigorous proof of Wiles [2], works aiming at a simple proof of the theorem continue to be reported constantly as reviewed by Schorer [3]. There are several reasons to this unceasing interest; some obvious while others slightly hidden. First of all, the theorem has such charming qualities of being simple, elegant, and manageable appearance that anyone half interested in mathematics cannot but feel like being capable of toying with it to some good end. The fact that the complete proof had not come for centuries and when it did it came in hundreds of pages has not disheartened the initiated ones at all. A very recent outcome of such steadfast efforts is due to Nag [4] [5] who reported a neat and short proof of Fermat's last theorem in a manner quite befitting to the general character of Diophantine equations. Of course, the proof is yet to stand against probable objections.

On the other hand, this attractive and amusing challenge has not been unanimously praised. Gauss (1777-1855), the most distinguished opponent, replied to Olbers in 1816 [1] that "I confess that Fermat's Theorem as an isolated proposition has very little interest for me, because I could easily lay down a multitude of such propositions, which one could neither prove nor dispose of." Likewise, Hilbert (1862-1943) was not keen on working Fermat's theorem as he explained why he was unwilling to do so [1]: "Before beginning I should put in three years of intensive study, and I haven't that much time to squander on a probable failure."

The present work in some manner sides with these reserved views and rather than tackling with the original equation suggests first a generalized form and then presents numerically obtained solutions to this variant of Fermat's last Diophantine equation. Thus, the quest to prove insolubility is reversed to find the forms and conditions that provide solutions. Accordingly, cubes are partitioned into three cubes; fourth powers into five different fourth powers, etc. In some cases geometric representations of solutions are offered as well as some conjectures concerning solubility of the general form for definite powers and terms.

\section{A Generalization of Fermat's Last Theorem}

Fermat's well-known last theorem states that the Diophantine equation

$$
z_{1}^{n}+z_{2}^{n}=z^{n}
$$

cannot be satisfied for positive integers $z, z_{1}, z_{2}$ when $n>2$. This theorem is generalized as follows.

Theorem 1. The Diophantine equation

$$
\sum_{i=1}^{m} z_{i}^{n}=z^{n}
$$

can have positive integer solutions $z_{i}, z \in \mathbb{Z}^{+}$only if $m \geq n$. The equation may not admit any solution at all for $n \geq 6$ despite the condition $m \geq n$ being satisfied.

The above theorem together with the statement concerning $n \geq 6$ is a con- 
jecture without proof hence should be regarded unsettled as was the case of Fermat's last assertion. Theorem 1 can be stated in a different form by allowing and admitting only rational solutions while restricting the solution domain between 0 and 1. Dividing (2) by $z^{n}$ gives

$$
\sum_{i=1}^{m} x_{i}^{n}=1
$$

where $x_{i}=z_{i} / z_{n}$ 's are all positive fractions or quotients, $x_{i} \in \mathbb{Q}$, confined to $0<x_{i}<1$. Accordingly, it is challenged to find rationals whose summation of the $n$th powers equals exactly to unity provided that $m \geq n$. The most important advantage of Equation (3) is that it provides visual observations of the solutions for $n=m=2$ and $n=m=3$ as demonstrated in $\$ 3$ and $₫ 4$, respectively.

\section{Second Power $n=2$ with Two Terms $m=2$}

We begin with the case for which the solutions are possible; namely, $n=m=2$ in (2) so that

$$
z_{1}^{2}+z_{2}^{2}=z^{2}
$$

For this case the solutions can be written as $z_{1}=k^{2}-l^{2}$ and $z_{2}=2 k l$ while $Z=k^{2}+l^{2}$, which represent all the primitive integer solutions or Pythagorean integer triples. Here, $k$ and $l$ are relatively prime and $k>l>0$. It is obvious that infinitely many solutions can be produced from each primitive solution by multiplying that particular solution by different integers.

Table 1 gives ten different primitive triples for $z_{1}^{2}+z_{2}^{2}=z^{2}$ and corresponding rational numbers satisfying $x_{1}^{2}+x_{2}^{2}=1$. Figure 1 depicts $x_{1}, x_{2}$ pairs of rationals satisfying $x_{1}^{2}+x_{2}^{2}=1$ as well as the pairs obtained by swapping $x_{1}$ 's and $x_{2}$ 's. To give an example, both $\left(x_{1}=3 / 5, x_{2}=4 / 5\right)$ and $\left(x_{1}=4 / 5, x_{2}=3 / 5\right)$ are plotted. Figure 1 is therefore symmetric about the line drawn at $45^{\circ}$ to both axes.

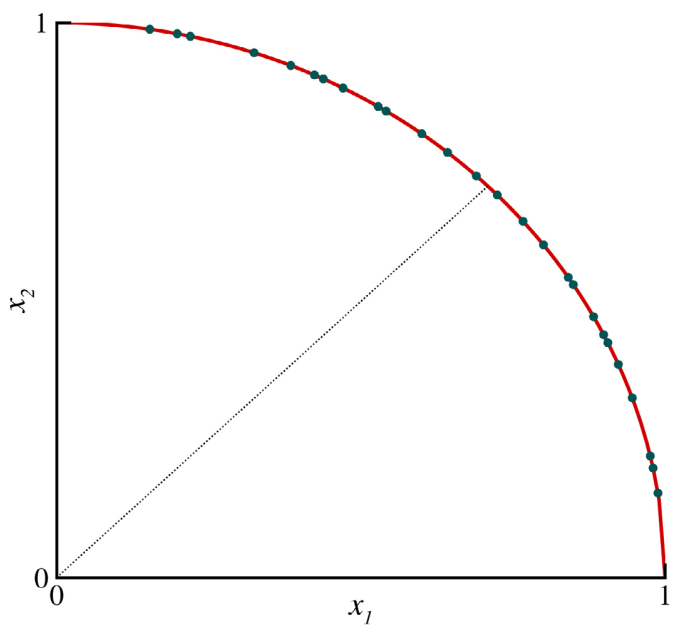

Figure 1. $x_{1}, x_{2}$ pairs of rationals (dots) satisfying $x_{1}^{2}+x_{2}^{2}=1$ as obtained from the integer solutions of $z_{1}^{2}+z_{2}^{2}=z^{2}$. 
Table 1. Ten primitive solutions of $z_{1}^{2}+z_{2}^{2}=z^{2}$ and corresponding rationals satisfying $x_{1}^{2}+x_{2}^{2}=1$.

\begin{tabular}{llr}
\hline$i$ & $z_{1}^{2}+z_{2}^{2}=z^{2}$ & $x_{1}^{2}+x_{2}^{2}=1$ \\
\hline 1 & $3^{2}+4^{2}=5^{2}$ & $(3 / 5)^{2}+(4 / 5)^{2}=1$ \\
2 & $8^{2}+15^{2}=17^{2}$ & $(8 / 13)^{2}+(12 / 13)^{2}=1$ \\
3 & $9^{2}+40^{2}=41^{2}+(15 / 17)^{2}=1$ \\
4 & $12^{2}+35^{2}=37^{2}$ & $(9 / 41)^{2}+(40 / 41)^{2}=1$ \\
5 & $13^{2}+84^{2}=85^{2}$ & $(12 / 37)^{2}+(35 / 37)^{2}=1$ \\
6 & $28^{2}+45^{2}=53^{2}$ & $(13 / 85)^{2}+(84 / 85)^{2}=1$ \\
7 & $36^{2}+77^{2}=85^{2}$ & $(28 / 53)^{2}+(45 / 53)^{2}=1$ \\
9 & $39^{2}+80^{2}=89^{2}$ & $(36 / 85)^{2}+(77 / 85)^{2}=1$ \\
10 & $20^{2}+99^{2}=101^{2}$ & $(39 / 89)^{2}+(80 / 89)^{2}=1$ \\
9 & & $(20 / 101)^{2}+(99 / 101)^{2}=1$ \\
\hline & &
\end{tabular}

Cases with more number of terms such as $m=n+1=3, m=n+2=4$, etc. have solutions too as anticipated. For instance, $1^{2}+4^{2}+8^{2}=9^{2}, 2^{2}+3^{2}+6^{2}=7^{2}$, and $3^{2}+4^{2}+12^{2}=13^{2}$ are just three primitive solutions of many more for $m=3$. Similarly, for $m=4$ we have $1^{2}+2^{2}+4^{2}+10^{2}=11^{2}$, $2^{2}+3^{2}+8^{2}+38^{2}=39^{2}, 3^{2}+4^{2}+8^{2}+44^{2}=45^{2}$, etc. It should be indicated that as $m$ gets larger compared to $n$ the number of primitive solutions within a given range of numbers gets more. For the same reason, while $n=m=4$ case reveals no solutions, increasing $m$ to $m=n+1=5$ results in a number of primitive solutions as presented in $\$ 5$. All such solutions can easily be obtained by a short and simple computer routine as given in the Appendix.

\section{Third Power $n=3$ with Three Terms $m=3$}

Setting $n=m=3$ in Equation (2) gives

$$
z_{1}^{3}+z_{2}^{3}+z_{3}^{3}=z^{3}
$$

A simple FORTRAN program given in the Appendix for $n=m=3$ is employed to seek integers satisfying Equation (5). The first ten primitive solutions obtained from a search covering integers in the range $1-100$ are listed in Table 2. Thus, while it is not possible to express the cube of a whole number as a summation of two cubes, it can be expressed as a summation of three or more cubes. The corresponding rational solutions satisfying $x_{1}^{3}+x_{2}^{3}+x_{3}^{3}=1$ are also given. Using the rational solutions Figure 2 plots $x_{1}, x_{2}, x_{3}$ triples on the cubic surface $x^{3}+y^{3}+z^{3}=1$. For a symmetric view $x_{2}, x_{1}, x_{3}$ triples are plotted too. 


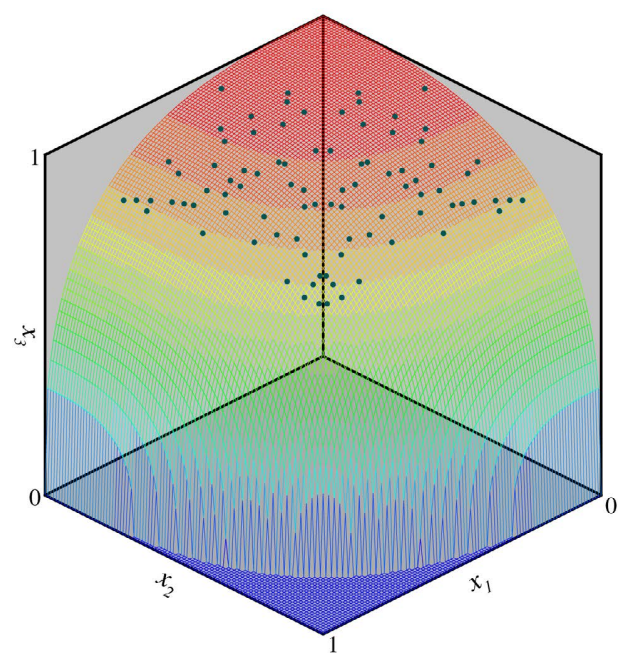

Figure 2. Rationals $x_{1}, x_{2}, x_{3}$ (dots) satisfying $x_{1}^{3}+x_{2}^{3}+x_{3}^{3}=1$ which correspond to the integer solutions of $z_{1}^{3}+z_{2}^{3}+z_{3}^{3}=z^{3}$. Points obtained by swapping $x_{1}$ and $x_{2}$ values are also shown for a symmetric view.

Table 2. Ten primitive solutions of $z_{1}^{3}+z_{2}^{3}+z_{3}^{3}=z^{3}$ and corresponding rationals satisfying $x_{1}^{3}+x_{2}^{3}+x_{3}^{3}=1$.

\begin{tabular}{llc}
\hline$i$ & $z_{1}^{3}+z_{2}^{3}+z_{3}^{3}=z^{3}$ & $x_{1}^{3}+x_{2}^{3}+x_{3}^{3}=1$ \\
\hline 1 & $1^{3}+6^{3}+8^{3}=9^{3}$ & $(1 / 9)^{3}+(6 / 9)^{3}+(8 / 9)^{3}=1$ \\
2 & $3^{3}+4^{3}+5^{3}=6^{3}$ & $(3 / 6)^{3}+(4 / 6)^{3}+(5 / 6)^{3}=1$ \\
3 & $2^{3}+17^{3}+40^{3}=41^{3}$ & $(2 / 41)^{3}+(17 / 41)^{3}+(40 / 41)^{3}=1$ \\
4 & $3^{3}+10^{3}+18^{3}=19^{3}$ & $(3 / 19)^{3}+(10 / 19)^{3}+(18 / 19)^{3}=1$ \\
5 & $3^{3}+36^{3}+37^{3}=46^{3}$ & $(3 / 46)^{3}+(36 / 46)^{3}+(37 / 46)^{3}=1$ \\
7 & $4^{3}+17^{3}+22^{3}=25^{3}$ & $(4 / 25)^{3}+(17 / 25)^{3}+(22 / 25)^{3}=1$ \\
8 & $6^{3}+32^{3}+33^{3}=41^{3}$ & $(6 / 41)^{3}+(32 / 41)^{3}+(33 / 41)^{3}=1$ \\
9 & $7^{3}+14^{3}+17^{3}=20^{3}$ & $(7 / 20)^{3}+(14 / 20)^{3}+(17 / 20)^{3}=1$ \\
10 & $7^{3}+54^{3}+57^{3}=70^{3}$ & $(7 / 70)^{3}+(54 / 70)^{3}+(57 / 70)^{3}=1$ \\
& $11^{3}+15^{3}+27^{3}=29^{3}$ & $(11 / 29)^{3}+(15 / 29)^{3}+(27 / 29)^{3}=1$ \\
& &
\end{tabular}

An interesting feature of primitive solutions is their clustering in a band of surface region in the upper part of the cubic surface.

Similar to the second power case, solutions are possible for the third power case when $m=4,5, \cdots$. Some computational results are $1^{3}+5^{3}+7^{3}+12^{3}=13^{3}$ and $2^{3}+3^{3}+8^{3}+13^{3}=14^{3}$ for $m=4$ and $1^{3}+2^{3}+4^{3}+12^{3}+24^{3}=25^{3}$ and $2^{3}+3^{3}+5^{3}+51^{3}+76^{3}=83^{3}$ for $m=5$. 


\section{Fourth Power $n=4$ with Four $m=4$ and Five Terms $m=5$}

Setting $n=m=4$ in Equation (2) gives

$$
z_{1}^{4}+z_{2}^{4}+z_{3}^{4}+z_{4}^{4}=z^{4}
$$

Running the program for $n=m=4$ yields no primitive integer solutions for the range 1 - 150. The range could not be increased further due to restricted machine capability of operating large numbers. Nevertheless, from this particular and other computations we make a tentative inference that if no solution is found in the range $1-100$ it is unlikely to be any solution at all. Accordingly we now increase the number of terms to $m=n+1=5$ so that

$$
z_{1}^{4}+z_{2}^{4}+z_{3}^{4}+z_{4}^{4}+z_{5}^{4}=z^{4}
$$

for which the solutions are obtained by using the second program given in the Appendix. Table 3 lists ten primitive solutions of Equation (7) computed by trying first 100 integers. Corresponding rational quantities $x_{1}, x_{2}, x_{3}, x_{4}, x_{5}$ satisfying $x_{1}^{4}+x_{2}^{4}+x_{3}^{4}+x_{4}^{4}+x_{5}^{4}=1$ are not given for this case as it is not possible to draw a 5-D graphic.

\section{Fifth Power $n=5$ with Five $m=5$ and Six Terms $m=6$}

Setting $n=m=5$ in Equation (2) results in

$$
z_{1}^{5}+z_{2}^{5}+z_{3}^{5}+z_{4}^{5}+z_{5}^{5}=z^{5}
$$

The search program for $n=m=5$ gives no primitive integer solutions for the range 1 - 50. Again, the range could not be increased more because of machine limits. On the other hand, increasing the number of terms to $m=6$ and thus considering

$$
z_{1}^{5}+z_{2}^{5}+z_{3}^{5}+z_{4}^{5}+z_{5}^{5}+z_{6}^{5}=z^{5}
$$

gives just two primitive solutions shown in Table 4 for the 50 integers that could

Table 3. Ten primitive solutions of $z_{1}^{4}+z_{2}^{4}+z_{3}^{4}+z_{4}^{4}+z_{5}^{4}=z^{4}$.

\begin{tabular}{lc}
\hline$i$ & $z_{1}^{4}+z_{2}^{4}+z_{3}^{4}+z_{4}^{4}+z_{5}^{4}=z^{4}$ \\
\hline 1 & $1^{4}+2^{4}+12^{4}+24^{4}+44^{4}=45^{4}$ \\
2 & $1^{4}+8^{4}+24^{4}+36^{4}+38^{4}=45^{4}$ \\
3 & $2^{4}+13^{4}+32^{4}+34^{4}+84^{4}=85^{4}$ \\
4 & $4^{4}+21^{4}+22^{4}+26^{4}+28^{4}=35^{4}$ \\
5 & $4^{4}+42^{4}+48^{4}+51^{4}+78^{4}=85^{4}$ \\
6 & $6^{4}+48^{4}+66^{4}+67^{4}+78^{4}=95^{4}$ \\
7 & $8^{4}+24^{4}+38^{4}+68^{4}+73^{4}=85^{4}$ \\
8 & $8^{4}+33^{4}+56^{4}+92^{4}+98^{4}=115^{4}$ \\
9 & $10^{4}+35^{4}+52^{4}+60^{4}+80^{4}=89^{4}$ \\
10 & $12^{4}+18^{4}+24^{4}+27^{4}+42^{4}=45^{4}$
\end{tabular}


Table 4. Two primitive solutions of $z_{1}^{5}+z_{2}^{5}+z_{3}^{5}+z_{4}^{5}+z_{5}^{5}+z_{6}^{5}=z^{5}$.

\begin{tabular}{cc}
\hline$i$ & $z_{1}^{5}+z_{2}^{5}+z_{3}^{5}+z_{4}^{5}+z_{5}^{5}+z_{6}^{5}=z^{5}$ \\
\hline 1 & $4^{5}+5^{5}+6^{5}+7^{5}+9^{5}+11^{5}=12^{5}$ \\
2 & $15^{5}+16^{5}+17^{5}+22^{5}+24^{5}+28^{5}=32^{5}$ \\
\hline
\end{tabular}

be covered. Note that for a given range the number of primitive solution obtained has decreased dramatically compared to the previous cases despite the increase in the number of terms $m$.

Searching solutions for $n=6$ with $m=6,7,8,9$ terms gave no results for integers in the range $1-28$. Here, 28 was the largest integer the machine could handle in computing the sixth power cases. Similar numerical searches for $n=7$ likewise failed to produce any solution hence attempts for higher powers were abandoned.

\section{Concluding Remarks}

A generalized variant of Fermat's last Diophantine equation is proposed by increasing the number of terms $m$ in accord with the power of terms $n$ and a corresponding theorem is stated without proof. No primitive solutions could be found for $m<n$ as in the case for Fermat's last theorem $m=2<n$. Solutions become possible only if $m=n$ or $m>n$, as could be intuitionally expected. Equating $m=n$ is sufficient for ensuring integer solutions to the second and third power equations but $m=n+1$ is needed for the fourth and fifth power equations. Geometric representations are presented for the cases $n=m=2$ and $n=m=3$ by normalizing the equations and thus confining the solution domain between 0 and 1. Primitive solutions are determined numerically by scanning integers up to 100 and listed in tables. The new Diophantine equation divides a cube into three or more cubes, a fourth power into five or more fourth powers and a fifth power into six or more fifth powers. While the required number of terms $m$ increases with increasing powers $n$ for getting equations with solutions, the number of primitive solutions within a definite range gets smaller. Therefore, with the aid of computations it is conjectured that after a certain power $n \geq 6$ there should be no solutions at all irrespective of the increase in the number of terms $m>n$. This is obviously just a surmise without any proof and therefore undecided. Finally, the ancient character of the Diophantine equations tacitly dictates only natural number solutions but it might be more interesting and rewarding to seek both positive and negative numbers and complex integers as well.

\section{Conflicts of Interest}

The author declares no conflicts of interest regarding the publication of this paper. 


\section{References}

[1] O'Conner, J.J. and Robertson, E.F. (2021) McTutor History of Mathematics Archive. http://www-history.mcs.st-andrews.ac.uk

[2] Wiles, A. (1995) Modular Elliptic Curves and Fermat's Last Theorem. Annals of Mathematics, 141, 443-551. https://doi.org/10.2307/2118559

[3] Schorer, P. (2016) Is There a "Simple" Proof of Fermat's Last Theorem? Part 1: Introduction and Several New Approaches. http://occampress.com/

[4] Nag, B.B. (2019) On Fermat's Last Theorem. Journal of Advances in Mathematics and Computer Science, 34, 1-4. https://doi.org/10.9734/JAMCS/2019/v34i230211

[5] Nag, B.B. (2021) An Elementary Proof of Fermat's Last Theorem for Epsilons. Advances in Pure Mathematics, 11, 735-740. https://doi.org/10.4236/apm.2021.118048

\section{Appendix}

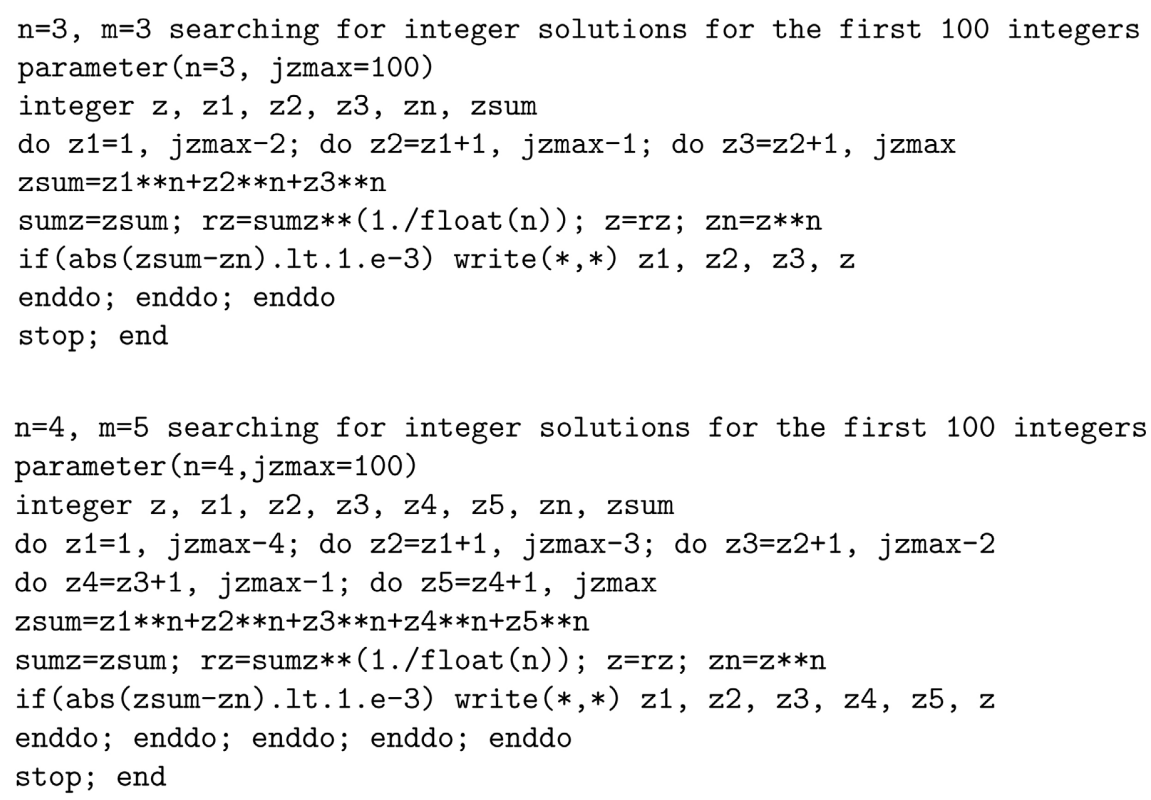

